\title{
Coproducción e Incidencia de la Sociedad Civil en la Política de Residuos Sólidos en Belém, Amazonia
}

Pinheiro de Andrade, Herbert Cristhiano; de Albuquerque Vasconcellos, Ana Maria; Vasconcellos Sobrinho, Mário; Pineda Nebot, Carmen

Coproducción e Incidencia de la Sociedad Civil en la Política de Residuos Sólidos en Belém, Amazonia Administração Pública e Gestão Social, vol. 12, núm. 2, 2020

Universidade Federal de Viçosa, Brasil

Disponible en: http://www.redalyc.org/articulo.oa?id=351562414011

Esta obra está bajo una Licencia Creative Commons Atribución-NoComercial-SinDerivar 3.0 Internacional. 


\title{
Coproducción e Incidencia de la Sociedad Civil en la Política de Residuos Sólidos en Belém, Amazonia
}

\author{
Coprodução e Incidência da Sociedade Civil na Política de Resíduos Sólidos em Belém, Amazônia \\ Civil Society Coproduction and Incidence on Solid Waste Policy in Belem, Amazon
}

Herbert Cristhiano Pinheiro de Andrade

Universidade Federal Rural da Amazônia, Brasil

Redalyc: http://www.redalyc.org/articulo.oa?

admherbert@yahoo.com.br

Ana Maria de Albuquerque Vasconcellos

Universidade da Amazônia, Brasil

Anamaria.vasconcellos@unama.br

\author{
Mário Vasconcellos Sobrinho \\ Universidade Federal do Pará - UFPA, Brasil \\ mariovasc@ufpa.br
}

Carmen Pineda Nebot

Universidad Autónoma de Madrid, España

camenpinedanebot@hotmail.com $\mathrm{id}=351562414011$

Recepción: 14 Agosto 2017 Aprobación: 17 Octubre 2018

\section{RESUMo:}

O artigo analisa a emergência de novas arenas e arranjos organizacionais para gestão de políticas públicas. Foca-se especificamente na gestão da política pública de resíduos sólidos na cidade de Belém (estado do Pará, Amazônia brasileira). Nesta cidade foi constituída uma rede de atores da sociedade civil denominada de Rede Social Nossa Belém, que protagoniza um projeto de sustentabilidade urbana que envolve a gestão de resíduos sólidos. Novos arranjos organizacionais têm sido formados envolvendo atores do Estado e da sociedade civil. O artigo busca entender em que medida essas novas arenas e arranjos têm apresentado resultados efetivos na gestão da política pública. Metodologicamente, a pesquisa é baseada no estudo de caso com abordagem qualitativa. $\mathrm{O}$ resultado da investigação mostra que emergiram arenas para acompanhamento de prestação de contas governamentais e para análise dos indicadores de eficiência da política pública. Constatou-se que há uma aproximação de arranjos coprodutivos na gestão da política pública.

PalaVras-CHaVE: Rede social, Arenas organizacionais, Coprodução.

\section{Resumen:}

El artículo analiza la emergencia de nuevas arenas y acuerdos organizacionales para la gestión de políticas públicas. Se focaliza específicamente en la gestión de la política pública de residuos sólidos en la ciudad de Belém (estado de Pará, Amazonia brasileña). En esta ciudad se creó una red de actores de la sociedad civil denominada Rede Social Nossa Belém que protagonizó un proyecto de sostenibilidad urbana que incluye la gestión de residuos sólidos. Como consecuencia del proyecto, se crearon nuevos acuerdos organizacionales incluyendo a actores del Estado y de la sociedad civil. El artículo busca entender en qué medida esas nuevas arenas y acuerdos han supuesto resultados efectivos en la gestión de la política pública. Metodológicamente, la investigación está basada en un estudio de caso con enfoque cualitativo. El resultado de la investigación muestra que emergieron arenas para el acompañamiento de la prestación de cuentas del gobierno y para el análisis de los indicadores de eficiencia de la política pública. Se constató que hay una aproximación de acuerdos coproductivos en la gestión de la política pública.

Palabras Clave: Red social, Arenas organizacionales, Coproducción.

\section{Abstract:}


The paper analyses the emergence of new arenas and organizational arrangements for public policy management. It focuses particularly on public solid waste policy management in the city of Belém (Pará state, Brazilian Amazon). In this city it was created a civil society network called Rede Nossa Belém (RNB) that carries out an urban sustainable project that involves solid waste management. New organizational arrangements have been built involving state and civil society actors for project's aims achievements. The article tries to understand to what extent the new arenas and arrangements have shown effective outcomes for public policy management. Methodologically, the research is based on a case study and qualitative approach. The research shows that arenas have emerged to follow government accountability and to analyse public policy efficiency indicators. It was identified an approximation of the concept of coproduction arrangements for public policy management.

KEYWORDS: Social network, Organizational arenas, Coproduction.

\section{INTRODUCCIÓN}

En Brasil, en un intento de superar tradicionales formas de relación entre sociedad civil y gobierno para la gestión de residuos sólidos y para el desarrollo de las ciudades se estableció la Ley n. ${ }^{\circ}$ 12.305/2010, la Política Nacional de Residuos Sólidos (PNRS). Esta determinó que los municipios brasileños deberían elaborar y entregar sus respectivos Planes de Gestión de Residuos Sólidos, imponiendo la erradicación de los llamados "vertederos". Hace bastante tiempo que en Brasil existe la recogida de basura, aunque solo pasó a ser considerada como un problema social durante la década de los 1990 (Pereira et al., 2014). La participación directa y activa del sector público, privado y de la sociedad en el proceso de elaboración e implementación de la política de residuos sólidos pasa a ser importante para definir sus directrices y objetivos, ya que para alcanzar la gestión adecuada es necesaria la participación colectiva en la elaboración del plan de gestión de los residuos (Cezar et al., 2014; Schommer, Bueno, Kunzler, 2010).

La ciudad de Belém tiene la producción per cápita más alta de residuos sólidos en la Amazonia brasileña y el segundo mayor vertedero de basura a cielo abierto de Brasil, llamado Aurá. Esta ciudad se encuentra en la mayor área metropolitana de la Amazonia y cuenta con aproximadamente 1.446.042 habitantes, un área territorial de $1059.458 \mathrm{~km}^{2}$ y 39 islas. La realidad vivida por su población remite a la idea de que estos residuos sólidos han sido un problema para la ciudad, en la medida en que su cadena productiva se encuentra desfragmentada. Sumado a eso, hace por lo menos 12 años que la postura tecnoburocrática de los gobiernos municipales ha colaborado para su distanciamiento de la sociedad civil organizada, en lo que respecta a la gestión de la cosa pública.

En este escenario, se creó en 2012 la Red Social Nossa Belém (RNB), que por medio del Programa Ciudades Sostenibles y de su Proyecto de Incidencia de la Sociedad Civil en la Sostenibilidad Urbana (PISCSU) busca coproducir el desarrollo territorial. La RNB es inter-religiosa, no partidista y representa a la sociedad civil con el objetivo de coproducir el bien público mediante el equilibrio de poder entre estado, mercado y sociedad civil, siendo corresponsable de la gestión de las políticas públicas. Esta red actúa en diversos frentes como la seguridad pública, la movilidad urbana y los residuos sólidos. En realidad, la RNB surge conectada a una iniciativa que se va expandiendo por América Latina. Se trata de la Red Latinoamericana de Ciudades y Territorios Justos, Democráticos y Sostenibles (RLCTJDC). Esta red social está ya presente en 10 países (Argentina, Bolivia, Brasil, Chile, Colombia, Ecuador, México, Paraguay, Perú y Uruguay) e inspira más de 70 iniciativas y movimientos ciudadanos en las ciudades. En Brasil, se denomina Red Brasileña por Ciudades Justas, Democráticas y Sostenibles y fue creada para movilizar a los ciudadanos sin adscripción política en acciones de monitoreo de indicadores de calidad de vida, promoción de la participación ciudadana, defensa de la transparencia y a favor del diálogo con los gobiernos locales y el sector privado.

Esta investigación se fundamenta en el análisis de las arenas y acuerdos organizacionales que surgen para la gestión de los residuos sólidos en Belém a partir de la RNB y de su Proyecto de Incidencia de la Sociedad Civil en la Sostenibilidad Urbana (PISCSU). Se busca analizar los actores que interactúan con la RNB social y las formas en las que ella moviliza a otros agentes y recursos. También destaca el espacio público en que 
estos actores se relacionan y en la forma en que está influyendo en la formación de acuerdos que implementa la PNRS.

Metodológicamente es un estudio de naturaleza cualitativa realizado desde junio de 2014 hasta junio de 2017. Para ello, fueran realizadas: (i) Búsqueda documental en los archivos del PISCSU; (ii) entrevistas semiestructuradas a 9 miembros claves de la RNB, entre ellos el presidente del Observatorio Social de Belém, organización que es secretaria ejecutiva de la RNB, 7 representantes del gobierno municipal, incluyendo a miembros de la Secretaria Municipal de Saneamiento (SESAN); (iii) observación participante en 8 reuniones del grupo de residuos sólidos de la RNB; y (iv) observación no participante en 4 eventos públicos.

Los eventos públicos fueron: (1) Panel de Cierre del Basurero de Aurá y la Implantación de la Recogida Selectiva en Belém, (2) Oficina de la Plataforma de Control de la Gestión Pública, (3) I Panel Ciudades Sostenibles y (4) Panel Dinero en la Basura: Desafíos y Oportunidades Pos Basurero de Aurá. En esos eventos hay que destacar la presencia de varios actores: catadores de residuos sólidos, fiscales y técnicos del Ministerio Público del Estado de Pará (MPPA). El MPPA es una institución dedicada a la defensa de los intereses sociales y no disponibles, tales como el derecho a la ciudadanía y de la ley y el sistema democrático. Los catadores son las personas que recogen los residuos las calles de la ciudad y lo revenden, básicamente, para chatarra. Algunos catadores trabajan en los vertederos a cielo abierto en situaciones insalubres y peligrosas. Destacamos que las observaciones realizadas y el guion utilizado en las entrevistas se vincularon a las categorías actores, arenas, acuerdos y coproducción.

Teóricamente, la gestión de la política pública es entendida en el sentido de "pluralidad de actores" que interactúan para gradualmente adecuar e incrementar las demandas sociales (Pressman \& Wildavsky, 1973). Así, en el caso específico de este artículo, se trata del movimiento multiactorial en la gestión de la PNRS en Belém. Esto porque esta política pública proclama la corresponsabilidad entre los actores para su consecución en el nivel municipal. De hecho, Amorim y Boullosa (2013) ya indicaban que la visión del monopolio estatal en la gestión de políticas públicas ha perdido espacio en un contexto marcado por la pluralidad de actores e intereses. Hay, según Amorim y Boullosa (2013), una reconfiguración de las lógicas de colaboración y coproducción de bienes y servicios públicos. Como apuntan Osborne, Radnor y Strokosch (2016) y Pestoff (2009), la coproducción es uno de los pilares de la reforma de las políticas públicas en todo el mundo y una respuesta al déficit democrático.

Los conceptos de arena y acuerdo son entendidos respectivamente como "espacio de comunicación para hacerlo juntos" (Cançado, Tenório y Pereira, 2011; Boullosa, 2013) y "una forma de articulación entre diferentes actores que actúan con participación y gestión social" (Salm, 2014). Este último es sugerido por Junqueira et al. (2012) como "acuerdo de gestión social", dado que se trata de una nueva modo de pensar el acuerdo y la política pública. De este modo, se parte de la opinión de que la interacción de y entre los actores en la arena de la política pública y en el acuerdo de gestión social puede convertirse en coproducción.

El artículo se divide en cuatro secciones además de esta introducción y las consideraciones finales. En la primera se realiza un debate teórico sobre la gestión de la política pública con el fin de mostrar el cambio de concepción del modelo centrado en el estado a un modelo de pluralidad de actores. La segunda sección, también teórica, trata de las arenas y los acuerdos organizacionales. En él se busca mostrar que existen nuevas configuraciones de arenas y acuerdos que han llevado a la coproducción de bienes y servicios públicos. La tercera presenta la Política Nacional de Residuos Sólidos y la utilización de nuevas arenas y acuerdos para su consecución. Esta sección también analiza en detalle el papel de la RNB en la gestión de la PNRS y se caracteriza como esta red contribuye a la coproducción del servicio público.

\section{Gestión de la Política Pública}

Charles Lindblom (1979) mostró el aspecto incremental en el análisis de la política pública. Este aspecto hace referencia a la toma de decisión construida por las interacciones entre los actores por la cual se ajusta 
gradualmente la política pública a una determinada realidad. Para Boullosa (2013), la política pública consiste en:

Un flujo de la historia de actores que se activan para el gobierno de problemas y/ o bienes considerados de pública relevancia a partir de una (re) comprensión individualizada, pero modelada socialmente, del mismo problema o bien que generó aquel flujo (p. 67).

La autora indica el problema o el bien público como las unidades analíticas centrales de la política para entender los flujos de acciones procedentes de una multiactorialidad activada por el interés en (ayudar a) gobernar un problema de pública relevancia. Desde esta perspectiva, el estudio de la política pública debe: (a) Definir el problema público; (b) comprender quienes componen la multiactorialidad; (c) identificar que es el interés o bien público; $(\mathrm{d})$ comprender la naturaleza de las acciones de gobierno del problema que produce el flujo (de políticas públicas).

Amorim y Boullosa (2013) alertan que:

El punto de vista del Estado como poseedor del monopolio de las políticas públicas, reduciendo al público a lo estatal, ha perdido espacio en un escenario marcado por la complejidad y pluralidad de actores y intereses implicados en los procesos de transformación social (p. 9)

Para estas autoras, las relaciones de enfrentamiento y antagonismo entre el Estado y la sociedad en el tratamiento de problemas de relevancia pública tiene que reconfigurarse en lógicas de colaboración y coproducción de bienes públicos.

La coproducción surge en la ciencia aproximadamente hace cuatro décadas, la idea fue acuñada por primera vez por Elionor Ostrom y su equipo de la Universidad de Indiana. El término fue utilizado en una serie de estudios sobre organización comunitaria y prestación de servicios de la policía de Chicago en la década de los 1970 (Ostrom et al., 1973). La expresión fue definida como un proceso a través del cual insumos utilizados para la producción de un bien o servicio incluyen a individuos que no están en la misma organización (Parks et al., 1981). Además de la percepción del ciudadano como insumo de producción, el significado de coproducción se vinculó a la concesión de servicios públicos, en una relación de igualdad y reciprocidad entre profesionales, las personas que utilizan los servicios, sus familias y los vecinos (Boyle \& Harris, 2009). En sus diversas formas, la coproducción se aplica a una diversidad de servicios que están siendo investigados y puestos en marcha en todo el mundo. Casos como los de la seguridad pública en Pakistán (Joshi \& Moore, 2004), de la merienda escolar en Dinamarca (Jakobsen, 2012), del transporte público en Ghana (Joshi \& Moore, 2004), de los cuidados infantiles en Suecia (Pestoff, 2009), del servicio de ecosistemas y bienestar en Tailandia (Lebel, Wattana, \& Talerngsri, 2015), del alcantarillado urbano (Bovaird, 2007) o de la infraestructura urbana (Ostrom, 1996) en Brasil, entre otros. Entre las actividades coproductivas Boyle y Harris (2009, p. 14) destacan que construir y fortalecer las redes sociales para el establecimiento de relaciones duraderas entre personas es una actividad coproductiva esencial para crear y transformar servicios públicos que implementen políticas públicas.

La red social se forma por múltiples articulaciones entre sujetos por el interés público y por situaciones a transformar. Estas articulaciones incluyen sujetos socialmente excluidos en nuevas formas de acción colectiva (Scherer-Warren, 2006). Las iniciativas buscan organizarse en redes sociales para ejercer una presión política sintonizada con los problemas de relevancia al amplificar estos problemas en la arena pública. La movilización de la red social hace que se coproduzca información para el control de la administración estatal y espacios no estatales de diálogo que presionen al gobierno en el combate a la corrupción y que sirva para atender las demandas de interés público (Schommer et al., 2015).

En el caso de los servicios públicos de residuos sólidos, Savas (1976) estudió el servicio de recogida de residuos sólidos de basura mixta domiciliaria y descubrió acuerdos coproductivos de auto-atención en diversas ciudades. Percy (1984) centralizó su estudio en la recogida y eliminación de residuos sólidos. Folz (1991) señaló que los esfuerzos del gobierno para incluir a los ciudadanos en el reciclaje de residuos sólidos correlacionan positivamente con la participación de los ciudadanos en el reciclaje. Cotterill et al. (2011) 
plantearon llevar a cabo un proyecto experimental para investigar los efectos de intervenciones que tuvieran como objetivo el aumentar la participación de los ciudadanos en áreas como el reciclaje de residuos.

Para ayudar a las organizaciones y comunidades a desarrollar la gestión, Governance Internacional elaboró un modelo guía con cinco etapas. La primera es mapear, en la que se identifican iniciativas existentes de coproducción y se explora el potencial para nuevos enfoques de coproducción. La segunda es focalizar, concentrarse en las actividades con mayor impacto en la definición de prioridades por medio de una evaluación de opciones. La tercera son las personas, la coproducción requiere que las personas estén dispuestas y sean capaces de coproducir y los representantes políticos locales desempeñen un papel positivo en la movilización de los vecinos. La cuarta es el mercado, para provocar un cambio de comportamiento en el compromiso de las comunidades locales. La quinta consiste en evolucionar, dentro y fuera de la organización y de la comunidad local.

El debate sobre la redefinición del espacio estatal para el público incluye la construcción de nuevos acuerdos que tengan como elemento innovador el establecimiento de nuevas formas de articulación entre Estado, sociedad civil y mercado, con la inclusión de nuevos actores en la gestión de la política pública en el nivel local (Farah, 2001). Los nuevos acuerdos coproductivos en políticas públicas surgen para discutir asuntos públicos en el espacio público donde se produce la relación entre la sociedad y el Estado en el proceso de reforma de la acción estatal. En vista de eso, la coproducción con participación social voluntaria y consciente se convierte en una oportunidad para el desarrollo de la ciudadanía y la diminución de las desigualdades sociales (Klein Jr. et al., 2012). La fuerza social en el interior de la coproducción, en detrimento de la formación de la opinión pública, según Bier et al. (2010), hace posible que los conflictos clarifiquen los intereses de los actores y representantes sobre la gestión de la política pública.

Por tanto, esa forma de gestión puede ser entendida como una forma de relación duradera de igualdad e reciprocidad entre actores, protagonizada por redes sociales compuestas por personas dispuestas y capaces de redefinir la arena de la política pública y construir nuevas formas de articulación, mediante acuerdos, que incluyan a nuevos actores en el proceso de gestión de las políticas públicas y reforme la acción estatal en el ámbito de la provisión de servicios públicos. Este proceso implica la participación directa de los ciudadanos en el esclarecimiento y superación de los conflictos en favor del interés público y del bien común.

\section{Arenas y Acuerdos de Gestión de la Política Pública}

En un intento inicial de presentar las opiniones sobre arenas y acuerdos utilizados en este trabajo, se parte de la opinión de que la arena de gestión de la política pública puede ser estatal o no. Tenório (1998) señala que la arena es un espacio de lucha por derechos. Esa comprensión de espacio de lucha es reforzada por Andion (2012), en la medida en que la autora hace alusión al espacio en el cual diferentes grupos de intereses se encuentran y se relacionan.

Por tanto, cuando actores sociales actúan en nombre del interés público lo hacen en el contexto de una específica arena de políticas públicas. Sus acciones recaen con mayor fuerza sobre la conformación de la arena de policy que sobre la (larga) esfera pública (Boullosa, 2013, p. 78).

En este punto, la coproducción, influida por la fundamentación Habermasiana, concibe al espacio público “como arena abierta a la discusión, en un proceso dialógico, de los diferentes grupos sociales" (Mattia $\&$ Zappellini, 2014, p. 586).

Sobre el acuerdo, se parte de la opinión de que se refiere a la interacción de actores que ocurre por medio de redes y socios u otros acuerdos organizacionales o societarios de los cuales participa el ciudadano (Salm, 2014). El acuerdo es construido por un proceso que articula una diversidad de actores y agentes en el sentido de una mayor aproximación para la obtención de acciones específicas. En los acuerdos, los diversos actores realizan actividades conjuntas de gestión de la política pública y esas actividades son entendidas como acciones (públicas y colectivas) (Lelieveldt et al., 2009; Salm \& Silva, 2015). El acuerdo coproductivo puede ser 
entendido como una forma de aproximar el Estado y la sociedad a la producción de bienes y servicios públicos que busquen el bien común, ofreciendo a la sociedad la posibilidad de participar en la definición de lo que constituye en cierto contexto un bien público y de las prioridades en términos de bienes y servicios públicos a construirse colectivamente. Los tipos de acuerdos coproductivos de los servicios públicos que tienen en cuenta la utilización del usuario y de la comunidad se caracterizan por una amplia gama de actividades de servicios que pueden ser incluidas en la coproducción. Se identificaron a partir de Bovaird y Loeffler (2013) e International (2016): Cocomisionamiento, codiseño, coprestación y coevaluación, entre otros.

En el ámbito de acuerdos coproductivos de planificación con participación de las redes sociales, Lelieveldt et al. (2009) argumentan que la implicación de organizaciones cívicas como actores políticos en la gestión de problemas públicos, culmina en la coproducción de un plan de resolución de problemas específicos del barrio. La red social, para afrontar los problemas y elaborar políticas, tiene que desarrollar la capacidad de dos tipos de contactos. El primer contacto es con políticos y miembros del gobierno. El segundo contacto es con socios, con el fin de movilizar recursos locales para la acción conjunta. Las redes sociales tienen más capacidad de movilizar a otras organizaciones para la acción colectiva y tienen más posibilidades de ser movilizadas por otros (Lelieveldt et al., 2009).

El codiseño de servicios públicos va más allá de la consulta al usuario, se trata de aportar la experiencia de los usuarios y de sus comunidades en el diseño de los servicios públicos (Bovaird \& Loeffler, 2013). La perspectiva del usuario y de la comunidad provoca muchas veces la innovación del servicio. El codiseño indica un enfoque estructurado para aprovechar las mejores ideas de las personas que terminaran por usar y hacer suyo el servicio (Governance, 2016). Incluye experiencia con el servicio, exploración de la creatividad, experimentación de las innovaciones, desarrollo y prueba de los prototipos por la comunidad y evaluación continua del servicio. En algunos casos se produce la experimentación del prototipo del servicio, para luego, criticarlo y empezar a discutir formas de mejorarlo. La co-assess de los servicios públicos se dividen en cocontrol y coevaluación de los servicios (Bovaird \& Loeffler, 2013). La coevaluación incluye a ciudadanos que trabajan al lado de profesionales y gestores públicos para ayudar a las organizaciones a entender mejor como perciben ellas los servicios. Eso presupone que una mejor calidad del servicio puede alcanzarse por medio de la coproducción colectiva, dado que también promueve una mayor transparencia y prestación de cuentas (Pestoff, 2009).

En la literatura brasileña, han surgido experiencias de coplanificación, coplanificación de la gestión, cocontrol social y acuerdos de inserción social. La coplanificación es un acuerdo coproductivo democrático y participativo que busca la construcción de un plan, su configuración incluye espacios de gobernanza, acciones de sensibilización y movilización de la sociedad en favor de la elaboración de planes, diagnósticos, predicciones, controles y evaluaciones (Mattia, 2014). Asimismo en el acuerdo de planificación, se identifica también una experiencia de coplanificación de gestión que incluye interacción, diálogo y decisiones compartidas y responsabilidad entre representantes gubernamentales y de la sociedad civil.

En un enfoque sociopolítico sobre coproducción, Schommer et al. (2015) han generado conocimiento de los acuerdos de coproducción de la información y del control sobre la administración pública. Los autores constataron que el foco analítico de la coproducción como prestación de cuentas se caracteriza por la multiplicidad de interacciones entre representantes públicos y la comunidad. En este caso, la sociedad civil se moviliza y conecta con el gobierno para activar el sistema de prestación de cuentas y alcanzar mejores resultados en términos de información, justificación, recompensa y castigo. Las informaciones y el control basados en la prestación de cuentas sirven como apertura para el diálogo y la coproducción y crean la posibilidad de canales de comunicación entre el control institucional y el control social. Sobre los acuerdos de control social compartido entre la administración pública y la comunidad en la implementación de políticas públicas, Magalhães y Souza (2015) analizan una experiencia de cocontrol social y afirman que "en la perspectiva del control social compartido entre la administración pública y la comunidad hay una gestión compartida, donde la comunidad es llamada a participar de la creación e implementación de las políticas públicas en torno al interés colectivo" (Magalhães \& Souza, 2015, p. 152). 
La articulación de múltiples actores, en la territorialidad municipal urbana de la gestión de la política pública requiere nuevos acuerdos de desarrollo local que permitan la distribución del poder y el fortalecimiento de la democracia (Souza et al., 2016), así como espacios de deliberación abiertos a la sociedad civil y a la ciudadanía, junto a nuevos procesos de coproducción. La posibilidad de involucrar a los ciudadanos en la acción pública, en un nuevo marco institucional contra hegemónico de un problema público, tiene como elemento central la coproducción (Dente \& Subirats, 2014). Sin embargo, la coproducción va más allá de la incidencia en la política pública en los espacios participativos de consulta. Tiene que ver con la manera de aumentar la capacidad de producción de valor público (Subirats, 2015). "Es así que la participación debería superar el simple espacio de consulta (buzón de demanda) para avanzar hacia un espacio deliberativo que genere procesos de coproducción de bienes y servicios públicos" (Zurbriggen \& Lago, 2014, p. 344). Ante lo expuesto, fue posible identificar categorías de análisis de la gestión de la política pública y construir una propuesta de enfoque teórico-metodológica que ayude a la discusión de los resultados de esta investigación. Las categorías son descritas en el cuadro 1.

\section{CUADRO 1}

Categorías de análisis del proceso de gestión

\begin{tabular}{|c|c|c|}
\hline Categoría & Descripción & Autores \\
\hline Actores & $\begin{array}{l}\text { Participación de diversos } \\
\text { actores sociales, estatales, } \\
\text { gubernamentales, políticos, } \\
\text { entre otros, en el proceso } \\
\text { productivo. Ellos son } \\
\text { coproductores en la medida en } \\
\text { que su capacidad contribuye } \\
\text { sustancialmente para la } \\
\text { producción del bien público. }\end{array}$ & $\begin{array}{l}\text { Schommer, Bueno y Kunzler } \\
\text { (2010); Bovaird y Loeffler, (2013); } \\
\text { International (2016); Frieling, } \\
\text { Lindenberg y Stokman (2014); } \\
\text { Subirats, (2015). }\end{array}$ \\
\hline $\begin{array}{l}\text { Arena de la } \\
\text { Política } \\
\text { Pública }\end{array}$ & $\begin{array}{l}\text { Espacio de gobernanza pública } \\
\text { el cual representa un lugar de } \\
\text { interacción para el diálogo entre } \\
\text { coproductores y que contribuye } \\
\text { a clarificar conflictos y a } \\
\text { construir soluciones. }\end{array}$ & $\begin{array}{l}\text { Mattia y Zappellini (2014); Bovaird } \\
\text { y Loeffler (2013); Mattia (2014); } \\
\text { Salm y Silva (2015); Souza et al. } \\
\text { (2016); Zurbriggen, y Lago (2014). }\end{array}$ \\
\hline Acuerdos & $\begin{array}{l}\text { Articulación entre } \\
\text { coproductores con una } \\
\text { finalidad específica } \\
\text { (planificación, gestión, control, } \\
\text { etc.) en el cual hay colaboración } \\
\text { en el trabajo conjunto } \\
\text { (transparencia, prestación de } \\
\text { cuentas, control sobre la } \\
\text { administración pública). }\end{array}$ & $\begin{array}{l}\text { Bovaird y Loeffler (2013); Perry y } \\
\text { Christensen (2015); OStrom } \\
\text { (1996); Parks et al. (1981); Pestoff } \\
\text { (2006); (Pestoff, 2009); Schommer } \\
\text { et al. (2015); Magalhães y Souza } \\
\text { (2015). }\end{array}$ \\
\hline Coproducción & $\begin{array}{l}\text { Gestión de la política pública } \\
\text { que culmina en producción } \\
\text { pública de bienes y servicios de } \\
\text { interés público. }\end{array}$ & $\begin{array}{l}\text { Dente y Subirats (2014); } \\
\text { Zurbriggen, y Lago (2014); } \\
\text { Schommer et al. (2015). }\end{array}$ \\
\hline
\end{tabular}

En este proceso de gestión, los actores sociales comprometidos se movilizan para interactuar con diversos actores, entre ellos el gobierno, y por medio de la arena política, dialogan, deliberan y deciden, así como dinamizan la formación de nuevos acuerdos democráticos y horizontales con consecuencias en la coproducción de bienes y servicios públicos. 


\section{La Política Nacional de Residuos Sólidos y la Red Nossa Belém en la gestión de la Política de Residuos Sólidos}

La PNRS considera corresponsables de la gestión de la política pública a los catadores de materiales reutilizables y reciclables y sus formas de asociación. Los catadores de residuos sólidos son las personas que en Brasil viven de la recogida y selección de la basura. Además de ser mencionadas las cuestiones relacionadas con la pobreza y la exclusión, los catadores organizados en sus movimientos sociales buscan la ciudadanía, fortalecer su identidad profesional (Carmo, 2011) e influir en las políticas públicas.

La gestión de la PNRS aconseja la relación entre Sociedad Civil y Gobierno Municipal y representa una oportunidad de coproducción de: (1) Servicio público de recogida selectiva de residuos sólidos previamente separados según sus características o composición; (2) control social que garantizan a la sociedad información y participación en los procesos de formulación, implementación y evaluación de las políticas públicas relacionadas con los residuos sólidos; (3) plan municipal de gestión integrada de residuos sólidos con diagnóstico, indicadores de desempeño, definición de corresponsabilidades y priorización de programas y acciones; (4) administración de residuos sólidos ejercida, directa o indirectamente, en las etapas de recogida, transporte, traslado, tratamiento y destino final ambientalmente adecuado de los residuos sólidos y disposición final ambientalmente adecuada de los desperdicios. Las directrices de la PNRS señalan diversas finalidades para la articulación entre los atores, entre las que implican a la sociedad civil y al gobierno municipal se identifican (cuadro 2).

\section{CUADRO 2}

Gestión de la Política Nacional de Residuos Sólidos

\begin{tabular}{|c|c|}
\hline Finalidades & Oportunidades \\
\hline $\begin{array}{l}\text { Coplanificación } \\
\text { de la gestión }\end{array}$ & Plan Municipal de gestión integrada de residuos sólidos \\
\hline \multirow[t]{2}{*}{ Cogestión } & Gestión integrada de residuos sólidos \\
\hline & Administración de residuos sólidos \\
\hline \multirow[t]{2}{*}{ Cocontrol } & Control Social de la Política Nacional de Residuos Sólidos \\
\hline & $\begin{array}{l}\text { Control Social del Plan Municipal de gestión integrada de residuos } \\
\text { sólidos }\end{array}$ \\
\hline \multirow{2}{*}{$\begin{array}{l}\text { Coprestación de } \\
\text { servicios } \\
\text { públicos }\end{array}$} & Servicio de recogida selectiva \\
\hline & $\begin{array}{l}\text { Servicio público de limpieza urbana y de manejo de residuos } \\
\text { sólidos }\end{array}$ \\
\hline $\begin{array}{l}\text { Coproducción de } \\
\text { la inclusión } \\
\text { social }\end{array}$ & $\begin{array}{l}\text { Inclusión socioproductiva de catadores de materiales reutilizables } \\
\text { y reciclables }\end{array}$ \\
\hline $\begin{array}{l}\text { Coproducción de } \\
\text { la Educación } \\
\text { Ambiental }\end{array}$ & Formación de ciudadanos \\
\hline
\end{tabular}

En el ámbito de la gestión de la PNRS desde la perspectiva municipal, presenta el plan municipal de gestión integrada de residuos sólidos y los planes de administración de residuos sólidos, garantizando su amplia publicidad y el control social en su formulación, implementación y operacionalización.

El abandono del gobierno municipal de Belém de la gestión del basurero de Aurá lo transformó en un basurero a cielo abierto, el conflicto entre el gobierno municipal y los catadores por la acción del gobierno para poner fin a las actividades en esta área y la sospecha de irregularidades en la contratación de una empresa para realizar la recogida selectiva culminó con acciones de la sociedad civil para la gestión de la Política Nacional de Residuos Sólidos y para prevenir la continuación de la situación ilegal. La RSNB, creó arenas de interacción de la política pública y se centró en la toma de decisiones. Estos espacios fueron llamados eventos públicos. Varios actores participaron en estos espacios. En ellos conversaron sobre los indicadores de los resultados 
de esta política pública y tomaron decisiones conjuntas sobre las trayectorias de gestión de residuos sólidos de la ciudad. La capacidad de incidencia de RNB influyó en la contratación de los catadores organizados en cooperativas para prestar el servicio de recogida selectiva municipal. En este sentido, la gestión se produce por la interacción entre los actores, yendo el protagonismo de la red social más allá de una experiencia consultiva y deliberativa. Sin embargo, además de la inclusión socioproductiva de los catadores, hay otros desafíos a la sociedad civil. Estos incluyen la reducción de la producción de residuos sólidos, vigilar los recursos públicos para los contratos de transporte de residuos y repensar críticamente el consumo.

Inicialmente el gobierno municipal no hizo ningún esfuerzo para incluir a los catadores de residuos sólidos en la recogida selectiva. Sin embargo, existía un compromiso firmado por el alcalde durante la campaña electoral de 2012 con el Programa Ciudades Sostenibles (PCS) que creó una oportunidad para que la RNB fuera un input del proceso de gestión. Otro punto fue la suspensión por el MPPA de la contratación de una empresa externa para realizar la recogida selectiva que dio como resultado una propuesta del MPPA de un Término de Ajuste de Conducta (TAC) firmado por el Ayuntamiento de Belém, comprometiéndose con acciones en la política de residuos sólidos.

La relación entre la RSNB y el Gobierno Municipal estuvo basada en la sinceridad, que puede apreciarse en la presentación por el Director del Departamento de Residuos Sólidos (DRS) de la SESAN de los indicadores de residuos sólidos del PCS. En esta arena, interacciones entre los interlocutores se dan por la discusión sobre cómo mejorar la ciudad como señalan Mattia y Zappellini (2014). Materializada en un Panel de Ciudades Sostenibles, el director abordó las conquistas y desafíos de la cadena de residuos sólidos de la Región Metropolitana de Belém, presentó el resultado de los indicadores de la ciudad y explicó las metas alcanzadas y no alcanzadas por el gobierno municipal. En una parte de la entrevista, como se puede ver a continuación, indicó la necesidad de una relación de sinceridad en la arena.

"Y lo que la gente necesita identificar, y quedó muy claro en el escrito, es que el objetivo de la presentación, y lo que la gente espera del poder público, es la sinceridad. Es la sinceridad de decir si no se está consiguiendo, decir los avances que se obtuvieron, los avances que ocurrieron los hemos dicho. Y también reconocer las limitaciones, por lo tanto lo que la gente espera del poder público es exactamente esa sinceridad de decir los desafíos que hay que enfrentar" (Miembro de la Red Nossa Belém).

La relación entre el gobierno municipal y los catadores de residuos sólidos es conflictiva en relación a la opinión sobre la PNRS, en la forma de apoyo que la alcaldía ofrece a las cooperativas y asociaciones de catadores de materiales reciclables, en la remuneración sobre el trabajo que el catador realiza y en la forma de contratación de las organizaciones de catadores para la realización de la recogida selectiva, como se puede ver por las palabras del catador. La RSNB demuestra ser una fuerza social como señalan Bier et al. (2010), pues es capaz de explicar los intereses de los actores y agentes sobre la política pública.

\footnotetext{
"Nosotros estamos intentando organizarnos con todas las actividades, porque si hoy yo vengo para aquí fue con el dinero que saque del material de allí atrás, veinte centavos, cincuenta centavos que fui acumulando... entonces está bien la forma en que el gobierno se moviliza para eso, para cerrar como usted mismo dice, garantizar un camión para aquella cooperativa no es solución, fue retirado el camión y en una reunión con el alcalde, fue cerrado, fue firmado un documento, que no se va a contratar con los de antes pero la gente va a intentar una contrapartida para intentar minimizar vuestra situación, garantizando una balsa para transportar nuestro material, garantizando un camión para transportar ese material que va a ser vendido, y que no está ocurriendo" (Catador de residuos sólidos).
}

El gobierno municipal destacó que el movimiento de catadores debía ser una empresa, y que los catadores puedan hacer en esta organización una gestión administrativa y contable profesional, como requisito para una relación más próxima con la alcaldía. Lo que indica una presión del gobierno municipal para la "empresarización" de los movimientos de catadores, y consecuentemente una penetración en la lógica de la tercerización.

Se observa en el argumento de Boyle y Harris (2009) sobre relaciones de igualdad y reciprocidad el consenso entre los catadores de la falta de reciprocidad del gobierno en el servicio de recogida selectiva. En la 
arena, la red social funciona como un moderador para establecer una relación de igualdad de participación entre el gobierno municipal y los catadores de residuos sólidos.

La RSNB actúa con trabajo voluntario en la organización de eventos públicos lo cual induce a los participantes de la red al debate y a no ver al gobierno como único responsable del desarrollo de ciudades justas y sostenibles. Actuando de manera propositiva para la mejora del servicio de recogida selectiva, movilizando personas para el control social de los recursos públicos destinados a contratos de la alcaldía para actuaciones relacionadas con los residuos sólidos.

La RNB por medio del PISCSU pudo coproducir la incidencia política. El PISCSU tiene como objetivo articular en red a los movimientos para que puedan ejercer el control social sobre las políticas públicas de movilidad urbana y de gestión de residuos sólidos.

"Existen muchas entidades que están haciendo buenas acciones, pero lo hacen de forma desarticulada y a veces hasta se solapan, haciendo lo mismo. La red tiene la finalidad de integrarlas... La red no es de ningún partido, es democrática y especialmente propositiva" (Miembro del Eslabón de Residuos Sólidos)

Ello tuvo como efecto el desarrollo de fuerzas coproductivas que incidieron en la contratación para el servicio de recogida selectiva de una organización de catadores de residuos sólidos, denominada Cooperativa de los Catadores de Materiales Reciclables (CONCAVES). Galli et al. (2014) afirman que la coproducción tiene un papel fundamental en las reglas de contratación de los servicios públicos, argumento que puede ser constatado. La CONCAVES coprodujo el servicio de recogida selectiva siendo identificada por el desarrollo de innovaciones en el servicio.

"Nunca contratamos con una cooperativa. La primera vez que ocurrió fue ese año y obviamente sabemos que existen una serie de problemas que deben superarse, pero el acto de cooperar, de contratar a una cooperativa, sea la que sea, abre un precedente para la contratación de otras cooperativas y para estimular el crecimiento y fortalecimiento del cooperativismo" (Director del Departamento Municipal de Residuos Sólidos).

La relación entre la RNB y el MPPA influyó en el debate de nuevos contratos públicos que abarquen a la ciudad como un todo y a las respectivas organizaciones de catadores localizadas en este territorio. Ese acuerdo, unido a la presión de los catadores de residuos sólidos ha incidido en las decisiones de compras del gobierno, según Galli et al. (2015).

El PCS tiene un conjunto de 300 indicadores de monitoreo del desarrollo de la ciudad. Basándose en esos indicadores son identificados los temas sobre coproducción. Los indicadores están agrupados en 12 ejes. Basándonos en esos ejes se realizó la búsqueda de iniciativas de coproducción existentes. Los indicadores comonitoreados del PCS fueron en este caso: Porcentaje de domicilios que disponen de recogida selectiva en relación al total de domicilios; porcentaje de catadores incluidos en el sistema de recogida selectiva, en relación al número total de catadores del municipio; media anual de residuos urbanos, en kilos, por habitante; porcentaje de residuos sólidos que es reciclado, en relación al total producido en la ciudad por año; porcentaje de basura de la ciudad que es depositada en depósitos por año, en relación al total de basura generada. Sobre la utilización de los indicadores, el fragmento de la entrevista que viene a continuación los presenta como una forma de cambio en la práctica actual de gestión pública y social.

"Existe una política nacional de residuos sólidos, existe un conjunto de obligaciones y eso no quiere decir que cada gestor a partir de la presentación de inversiones, únicamente de números, con decir que gastó un millón, dos millones, cien millones en una materia, signifique que dio resultados, su resultado es medido por medio del indicador, y a pesar de ser un nombre tan poco utilizado en la administración pública y en la sociedad, el indicador es una evidencia, es una señal de cómo está la situación" (Presidente del Observatorio Social de Belém).

Pese al esfuerzo de los representantes políticos locales de la RSNB para la incorporación de personas a la red, existe la necesidad de que más personas estén dispuestas y sean capaces de coproducir. No obstante, existen oportunidades para que los comprometidos puedan constituir de forma autónoma diversos eslabones de la red y, de ese modo, se corresponsabilicen de nuevas acciones conjuntas. 
La RNB protagonizó la realización de diversos eventos que demostraron la materialización del espacio público de acción de los coproductores. Mitlin (2008) llama coproducción de abajo a arriba las acciones del compromiso cívico de la sociedad civil. Al menos tres eventos fueron cruciales para la construcción colectiva de propuestas para el gobierno municipal. El primero se llamó "Cerramiento del Vertedero de Aurá y la implantación de la Recogida Selectiva en Belém” y sirvió para la construcción colectiva de estrategias orientadas al cerramiento del Vertedero de Aurá, de forma negociada con las personas que sobreviven de ello y la implantación de la recogida selectiva en Belém, estimulando la inclusión socio-productiva de los catadores, ya asegurada por la legislación.

El segundo fue el I Panel Ciudades Sostenibles, que hizo posible la divulgación de los indicadores de sostenibilidad del municipio, relacionados con cuestiones sobre los residuos sólidos, con un debate sobre los avances obtenidos y las oportunidades de mejora de la gestión del municipio de Belém. El tercero fue "Dinero en la Basura: Desafíos y Oportunidades después del Vertedero de Aurá”. En él se profundizó en el debate sobre la situación de funcionamiento del nuevo vertedero, se propusieron vías para ampliar la recogida selectiva en términos del área que abarcaría y el número de cooperativas contratadas y se discutieron las acciones del gobierno para la educación ambiental de reducción de producción de residuos por la población.

Se analizó que esos eventos se enmarcan en lo indicado por Mattia y Zappellini (2014) sobre espacio público pues en ellos existe interacción entre diversos participantes para la discusión de la acción de la política pública, en particular, sobre como cerrar las actividades del Vertedero de Aurá, implantar la recogida selectiva, controlar mediante indicadores el desarrollo de la ciudad en base a la inclusión de los catadores de residuos sólidos.

La codesign fue el tipo de acuerdo coproductivo identificado en la contratación de la cooperativa de catadores de residuos sólidos (CONCAVES) para la recogida selectiva. Un marco se dio en la Reunión de Trabajo para la Elaboración del Término de Referencia Ampliación y Operacionalización de la Recogida Selectiva en tres barrios del Municipio de Belém. En esta acción conjunta actuó de moderador el Observatorio Social de Belém, el cual administra ejecutivamente a la RNB.

Se puede ver que el MPPA, catadores de residuos sólidos y el gobierno municipal son los principales actores que interactúan con la RSNB. También se constata que la movilización social protagonizada por la RSNB se produce a través de la conexión de actores sociales comprometidos con el desarrollo de la ciudad. Sin embargo, son raros todavía los socios voluntarios.

El proceso de gestión surgió de los conflictos y el sistema para aplicar la política pública fue de arriba hacia abajo. La incidencia de la sociedad civil en los espacios de deliberación fue favorecida por las organizaciones civiles. La discusión se basa en la interpretación de la legislación. La interacción entre los actores engendró acuerdos coprodutivos mediados por la sociedad civil. Ellos amplifican la voz de actores históricamente excluidos de los espacios deliberativos, los catadores.

La existencia de redes sociales dirigidas al desarrollo local demuestra que ellas se estructuran para la gestión de la política pública. Ellas redefinen la arena por la relación con el gobierno municipal y con los demás implicados en la reforma de la acción estatal. La Red Social actúa en la arena como moderador en los conflictos de intereses y defensor de la supremacía del interés público.

Entre los diferentes tipos de acuerdos coproductivos identificados en la literatura, esta investigación revela que la arena contribuyó a la formación de acuerdos de gestión. En el proceso de diagnóstico compartido se construyen acuerdos de comonitoreamiento de indicadores, cocontrol social de los recursos públicos, con inclusión social. Este trabajo puede orientar a organizaciones sociales y gubernamentales en el proceso de gestión, necesitando del esfuerzo de sus representantes políticos locales (copromotor social) para movilizar personas, conectar causas y mediar en las discusiones de asuntos públicos. Esas son actividades claves para el desarrollo de la capacidad coproductiva. 


\section{Consideraciones Finales}

Los datos de la investigación muestran que la gestión puede ser entendida como una alternativa viable para lidiar con los conflictos y fallos de la administración pública. Sumado a eso, las observaciones sobre las arenas de diálogo se muestran como elementos importantes para la comprensión de esta gestión a partir del momento en que significan espacios de intermediación entre una política pública nacional y sus desarrollos a nivel local. Durante la investigación se observó que la incidencia de la sociedad civil en la toma de decisión colectiva en procesos de coproducción amplían las posibilidades del ejercicio de la ciudadanía en las políticas públicas, mediante el papel ciudadano creador y mediador de estos espacios y también articulador de acuerdos más inclusivos. Los datos de la investigación sugieren la coproducción como un proceso que se implica en la reforma de la acción estatal por medio de acuerdos de gestión pública local y desarrollo local.

La coproducción es una alternativa para que la sociedad civil pueda incidir en el rumbo de la política pública y con ello mejorar los servicios públicos prestados en su territorio. En un contexto de conflictos socioambientales en el territorio, algunos actores interactúan en el espacio público para dialogar, deliberar y tomar decisiones sobre sus problemas. La capacidad política coproductiva de los actores se revela fundamental para movilizar, mediar, conectar, equilibrar y democratizar interacciones descentralizadoras. Aún así, para la efectiva operacionalización de estas decisiones, se vio la necesidad de la formación de acuerdos con el fin de monitorear los indicadores de la política pública, proponer ajustes a los servicios públicos y sobre todo, testar la reforma del Estado.

El marco legal de la política pública y la existencia de instituciones de defesa de los derechos son partes integrantes de un proceso de gestión que empodera a actores históricamente excluidos. Sumado a eso, este trabajo pretende contribuir a ampliar las posibilidades del ejercicio de la ciudadanía, pues a ella cabe también la idealización de programas y proyectos que cambien a mejor, de forma articulada con el gobierno, la realidad colectiva.

Entre las limitaciones de la investigación está el periodo de tiempo del estudio, teniendo en cuenta que un estudio sobre la sostenibilidad urbana y los cambios importantes en los indicadores de impacto de una política pública de naturaleza compleja como la política nacional de residuos sólidos se producen ciertamente en un período largo tiempo. Otra limitación de esta investigación tiene que ver con la centralidad que se le ha dado a la RNB y a los principales actores que se relacionan con ella, pues se encontraron otros actores y acciones relacionados con esta política pública que no interactuaban directamente con esta red social.

Nuevas posibilidades de investigación surgen a partir de este trabajo, uno de ellos sería la profundización del estudio del proceso coproductivo, al ser éste dinámico y no lineal. La contribución de la coproducción para el desarrollo territorial, la dialogicidad en los espacios coproductivos, los tipos de incidencia de la sociedad civil en la toma de decisiones colectivas y la gobernanza pública en la coproducción de valor público son algunas cuestiones a ser exploradas.

\section{ReFERENCIAS}

Amorim, S., Boullosa, R. F. (2013). O estudo dos instrumentos de políticas públicas: uma agenda em aberto para experiências de migração de escala. Amazônia, Organizaçôes e Sustentabilidade, 2(1), 59-69.

Andion, C. (2012). Por uma nova interpretação das mudanças de paradigma na administração pública. Cadernos EBAPE.BR, 10(1), 01-19.

Bier, C. A., Feuerschütte, S. G., Schmitz, L. C., Bousfield, R., Simm, T. A., Pena, F. T. G. (2010). Sociedade, empresa e governo: Uma experiência de implementação de um novo paradigma de democracia participativa. Cadernos EBAPE. BR, 8(4), 580-599. doi: http://dx.doi.org/10.1590/S1679-39512010000400002

Bovaird, T. (2007). Beyond engagement and participation: User and community coproduction of public services. Public Administration Review, 67(5), 846-860. doi: 10,1111 / j.1540-6210.2007.00773.x 
Bovaird, T., Loeffler, E. (2013). We're all in this together: Harnessing user and community co-production of public outcomes. Birmingham, UK: University of Birmingham.

Boyle, D., Harris, M. (2009). The challenge of co-production. Londres, UK: New Economics Foundation.

Boullosa, R. F. (2013). Mirando ao revés nas políticas públicas: notas sobre um percurso de pesquisa. Revista Pensamento \& Realidade, 28(3), 67-84.

Cançado, A.C., Tenório, F. G., Pereira, J. R. (2011). Gestão social: reflexões teóricas e conceituais. Cadernos EBAPE.BR, 9(3), 681-703.

Carmo, M. S. (2011). As políticas de estimulação ao trabalho dos catadores no Rio de Janeiro como estratégia oriunda da valorização dos resíduos. Cadernos Gestão Pública e Cidadania, 16(58), 85-105. Recuperado de http://bibli otecadigital.fgv.br/ojs/index.php/cgpc/index

Cezar, L. C., Barbosa, T. R. C. G., Reis, M. C. T., Júnior, F. F. (2014). A publicização para além da espacio pública: Análise dos canais institucionais de participação da Política Nacional dos Resíduos sólidos. XXXVIII Encontro da EnANPAD.

Cotterill, S., Moseley, L. R. A., Smith, G., Stoker, G., John, C. W. P. (2011). Nudge, nudge, think, think: Using experiments to change civic behavior. Londres, UK: Bloomsbury Academic.

Joshi, A., Moore, M. (2004). Institutionalised co-production: Unorthodox public service delivery in challenging environments. Journal of Development Studies, 40(4), 31-49. doi: http://dx.doi.org/10.1080/0022038041000 1673184

Dente, B., Subirats, J. (2014). Decisiones públicas: Análisis y estudio de los procesos de decisión en políticas públicas. Barcelona: Colección Ariel Ciencias Sociales.

Farah, M. F. S. (2001). Parcerias, novos acuerdos institucionais e políticas públicas no nível local de governo. Revista de Administração Pública, 35(1), 119-144. Recuperado de http://bibliotecadigital.fgv.br/ojs/index.php/rap

Folz, D. H. (1991). Recycling solid waste: Citizen participation in the design of a coproduced program. State \& Local Government Review, 23(3), 98-102. Recuperado de http://www.jstor.org/table/4355016

Frieling, M. A., Lindenberg, S. M., Stokman, F. N. (2014). Collaborative communities through coproduction: Two case studies. The American Review of Public Administration, 44(1), 35-58. doi: 10.1177/0275074012456897

Galli, F., Brunori, G., Iacovo, F., Innocenti, S. (2014). Co-producing sustainability: Involving parents and civil society in the governance of school meal services. A case Study from Pisa, Italy. Sustainability, 6(4), 1643-1666. doi: $10.3390 /$ su6041643

International, G. 2016. Achieving citizes outcomes. Recuperado de http://www.govint.org/our-services/co-productio $\mathrm{n} /$.

Jakobsen, M. (2012). Can government initiatives increase citizen coproduction? Results of a randomized field experiment. Journal of Public Administration Research and Theory, 23(1), 27-54. doi: https://doi.org/10.1093 /jopart/mus036

Junqueira, F. P., Dias, S. L. F., Wanderley, M. B, Mendonça, P. (Org.). (2012). Gestão social: mobilizações e conexões. São Paulo: LCTE Editora.

Klein Jr, V. H., Salm, J. F., Heidemann, F. G., Menegasso, M. E. (2012). Participação e coprodução em política habitacional: Estudo de um programa de construção de moradias em SC. Revista de Administração Pública, 46(1), 25-48. Recuperado de http://bibliotecadigital.fgv.br/ojs/index.php/rap

Lebel, L., Wattana, S., Talerngsri, P. (2015). Assessments of ecosystem services and human well-being in Thailand build and create demand for coproductive capacity. Ecology and Society, 20(1). doi: http://dx.doi.org/10.5751 /ES-06527-200112

Lelieveldt, H., Dekker, K., Volker, B., Torenvlied, R. (2009). Civic organizations as political actors: Mapping and predicting the involvement of civic organizations in neighborhood problem-solving and coproduction. Urban Affairs Review, 45(1), 3-24. doi: 10.1177/1078087409332303

Lindblom, C. E. (1979). Still muddling, not yet through. Public Administration Review,39(6), 517-526. 
Magalhães, T. G., Souza, V. B. (2015). O Controle Social no Conselho Municipal do Idoso de Florianópolis: Uma análise a partir dos modelos de administração pública, dos modelos de coprodução dos serviços públicos e dos modos de gestão estratégica e social. Revista de Ciências da Administração, 1(3), 149-164. doi: http://dx.doi.or g/10.5007/2175-8077.2015v17nespp149

Mattia, C. (2014). A metodologia para a elaboração dos planos estaduais de cultura fomenta a coprodução do bem público? Politicas Culturais em Revista, 7(1), 100-118. Recuperado de https://portalseer.ufba.br/index.php/pc ulturais

Mattia, C. , Zappellini, M. B. (2014). Ética e coprodução de serviços públicos: Uma fundamentação a partir de Habermas. Cadernos EBAPE.BR, 12(3), 573-589. doi: http://dx.doi.org/10.1590/1679-395114417

Mitlin, D. W. (2008). With and beyond the state - co-production as a route to political influence, power and transformation for grassroots organizations. Environment \& Urbanization, 20(2), 339-360. doi: $10.1177 / 0956247808096117$

Osborne, S. P., Radnor, Z., Strokosch, K. (2016). Co-Production and the Co-Creation of Value in Public Services: A suitable case for treatment? Public Management Review, 18(5), 639-653. doi: Http:// dx.doi.org/10.1080/14719037.2015.1111927

Ostrom, E., Baugh, W.H., Guarasci, R., Parks, R. B., Whitaker, G. P. (1973). Community organization and theprovision of police services. Beverly Hills, BH: Sage Publications.

Ostrom, E. (1996). Crossing the great divide: Coproduction, synergy, and development. World Development, 24(6), 1073-1087. doi: http://dx.doi.org/10.1016/0305-750X(96)00023-X

Parks, R. B., Baker, P. C., Kiser, L., Oakerson, R., Ostrom, E., Ostrom, V., Wilson, R. (1981). Consumers as coproducers of public services: Some economic and institutional considerations. Policy Studies Journal, 9(7), 1001-1011. doi: 10.1111/j.1541-0072.1981.tb01208.x

Percy, S. L. (1984). Citizen Participation in the Co-production of Urban Services. Urban Affairs Review, 19(4), 431-446. Recuperado de http://journals.sagepub.com/doi/abs/10.1177/004208168401900403

Pereira, J. R., Carvalho, T. V., Cabral, E. H. S., Machado, R. T. M., Boas, A. A. V. (2014). Associações e Cooperativas de Catadores de Materiais Recicláveis: Perspectivas e dilemas em duas experiências do Sulde Minas Gerais. VI Encontro de Administração Pública da ANPAD.

Perry, J. L., Christensen, R. K. (2015). Handbook of Public Administration. John Wiley \& Sons.

Pestoff, V. (2009). Towards a paradigm of democratic participation: Citizen participation and co\#production of personal social services in Sweden. Annals of Public and Cooperative Economics, 80(2), 197-224. doi: 10.1111/ j.1467-8292.2009.00384.x

Pressman, J. L., Wildavsky, A. B. (1973). Implementation: How Grert Expectations in Washington are Dashed in Oakland, Or, why It's Amazing that Federal Programs Work at All. University of California Press.

Salm, J. F. (2014). Coprodução de bens e serviços públicos. In: BOULLOSA, Rosana de Freitas (org.) Dicionário para a formação em gestão social. Salvador: CIAGS/ UFBA, 42-44.

Salm, J. F., Menegasso, M. E. (2009). Os modelos de administração pública como estratégias complementares para a coprodução do bem público. Revista de Ciências da Administração, 11(25), 97-120. Recuperado de https://per iodicos.ufsc.br/index.php/adm

Salm, J. F., da Silva, M. H. (2015). A inserção social dos programas stricto sensu em administração das universidades públicas e a coprodução do bem público. Revista de Ciências da Administração, 17(41), 143-155. Recuperado de https://periodicos.ufsc.br/index.php/adm

Savas, E. S. (1976). Solid waste collection in metropolitan areas. In Elinor Ostrom, ed. The Delivery of Urban Services, Outcomes of Change. Beverly Hills, BH: Sage Publications.

Scherer-Warren, I. (2006). Das mobilizações às redes de movimentos sociais. Sociedade e Estado, 21 (1), 109-130.

Schommer, P. C., Rocha, A. C., Spaniol, E. L., Dahmer, J., Sousa, A. D. D. (2015). Accountability and co-production of information and control: Social observatories and their relationship with government agencies. Revista de Administração Pública, 49(6), 1375-1400. doi: http://dx.doi.org/10.1590/0034-7612115166 
Schommer, P. C., Bueno, R. U. H., Kunzler, R. (2010). Coprodução de serviços públicos e gestão de resíduos sólidos: Caminhos e obstáculos na experiência de um município catarinense. IV Encontro de Administração Pública e Governança da ANPAD.

Silva, E. T., Boeira, N., Salm, J.F, Menegasso, M. E. (2008). A Co-produção na Implementação da Política Pública de Prevenção e Erradicação do Trabalho Infantil: O Projeto Cata-vento. III Encontro de Administração Pública e Governança da ANPAD.

Souza, Z. B., Nabuco, A. L., Silva Filho, J. S., Ferreira, R. N., Almeida, M.C.M. (2016). Ação Pública e a construção de uma nova territorialidade urbana em Belo Horizonte (MG). Cadernos Gestão Pública e Cidadania, 21(70), 176-189. doi: http://dx.doi.org/10.12660/cgpc.v21n70.64338

Subirats, J. Políticas urbanas e innovación social: Entre la coproducción y la nueva institucionalidade - Criterios de significatividad. In Subirats, J., \& Bernardos, A. G. (2015). Innovación social y politicas urbanas en España: Experiencias significativas en las grandes ciudades.Icaria: Barcelona.

Tenório, F. G. (1998). Gestão social: uma perspectiva conceitual. Revista de Administração Pública, 32(5), 7-23.

Zurbriggen, C., Lago, M. G. (2014). Innovación y co-creación: Nuevos desafíos para las políticas públicas. Revista de Gestión Pública, 3(2), 329-361. Recuperado de http://www.revistadegestionpublica.cl/

\section{BY-NC-ND}

